approach is likely to be useful in practice. Unfortunately, the paper fails to look at the possible downsides of such a practice.

Potential adverse outcomes include short- and long-term stigma for the individual patient and loss of therapeutic relationship between the patient and clinician. These are likely to result in poorer services and longer periods of detention. The critical step in deciding whether to refer a patient to the criminal justice system will be the clinician's judgement of non-trivial violence. Good training can reduce lack of consistency but long-term follow-up and critical examination of this practice will ensure that adverse outcomes are kept to a minimum as we juggle to find the ethical balance here.

1 Wilson S, Murray K, Harris M, Brown M. Psychiatric in-patients, violence and the criminal justice system. Psychiatrist 2012; 36: 41-4.

Lin Sun Choong, consultant psychiatrist, Hadley Unit, Newtown Hospital, Worcester WR5 1JG, email: steve.choong@hacw.nhs.uk

doi: $10.1192 / p b .36 .5 .194 b$

\section{Prosecuting violent in-patients: the importance of staff attitudes}

The editorial by Wilson et al highlights important dilemmas faced by mental health professionals in relation to reporting violence perpetrated by mentally disordered patients. We welcome the proposals made by the authors, but unless there is a significant change in staff attitudes to reporting non-trivial violence perpetrated by psychiatric patients, progress in this area is unlikely to occur.

Our observation is underpinned by the results of two surveys which we carried out in a medium secure unit in Middlesbrough in 2006 and 2008. There were 80 incidents of assaults on staff by in-patients, the majority of incidents having been perpetrated by a minority (2006: 43 assaults, $n=10 / 100$; 2008: 37 assaults, $n=14 / 100$ ). Despite being a medium secure unit, the majority of assaults were perpetrated by patients detained under Part 2 of the Mental Health Act and by female patients. Only 10 incidents (12.5\%) were reported to the police, despite $70 \%$ of nursing staff being aware of the memorandum of understanding (www.cp3.gov.uk/publications/agencies/mounhs.html). We explored the attitudes of nursing staff using self-report attitude questionnaires (each of the 13 attitude statements measured on a 5-point Likert scale) to identify enablers or barriers to reporting incidents.

In both surveys, approximately a third of respondents feared that reporting incidents would result in a breakdown of therapeutic relationships with patients and a half feared reprisal from patients following reporting. In 2006, half of respondents considered being assaulted as an 'occupational hazard', but encouragingly this attitude was reported only by a quarter of respondents in 2008 . Although $84 \%$ of nursing staff understood that they had a 'right to report', a fifth believed that reporting incidents was a bureaucratic exercise without any benefits and for $60 \%$ the required reporting forms and procedures were difficult to complete. Staff were more likely to report incidents perpetrated by patients with personality disorder than those with other mental illness. About $20 \%$ of staff stated that they would only report incidents which resulted in physical injury. Only $40 \%$ believed that reporting incidents would strongly deter patients from re-assaulting. Some of these free-text comments capture the ambivalence in this area: 'I came to the nursing profession to help patients, not to be a punch bag'; 'I would report only if the assaults were due to "badness" not "madness"'; 'Disillusioned towards the police dealing with incidents'; 'Waste of time'; 'Zero tolerance should mean zero tolerance'.

In summary, whereas we acknowledge the value in developing robust policies, procedures and systems to address this important issue, significant progress in this area is unlikely to occur unless considerable efforts are made to shift attitudes of mental health professionals. Campaigns and systems to report and reduce violence are akin to taking a horse to water. Making a change will require a change of attitudes in relation to reporting violent incidents to the police. We propose that this can be achieved by discussing patient assaults in staff induction training, appraisal, supervision sessions and trust audits.

1 Wilson S, Murray K, Harris M, Brown M. Psychiatric in-patients, violence and the criminal justice system. Psychiatrist 2012; 36: 41-4.

Pratish B. Thakkar, consultant forensic psychiatrist, Tees, Esk and Wear Valleys NHS Foundation Trust, email: pratish.thakkar@tewv.nhs.uk; Ranjit Kini, clinical director, Primrose Programme, Tees, Esk and Wear Valleys NHS Foundation Trust.

doi: $10.1192 / p b .36 .5 .195$

\section{Discrimination between psychotropic and non-psychotropic treatment by patients}

Perecherla \& Macdonald ${ }^{1}$ state that they found no evidence that patients discriminated between psychotropic and nonpsychotropic treatment. Elsewhere, a lack of concordance with psychotropic medication has been reported to be as high as $75 \%$ over the course of a year. ${ }^{2}$ Although this may be on a par with adherence to non-psychotropic medications, there were significant factors which were not taken into consideration in Perecherla \& Macdonald's study.

Only patients who could communicate in English were included. This may have excluded patients from ethnic minority groups and other backgrounds, thereby ignoring their cultural and religious beliefs regarding medication. This surely must reduce the relevance of the results to populations with a significant proportion of ethnic groups. Further, the authors were unable to ascertain the duration of treatment in participants. This is an important factor as adherence improves with development of insight. ${ }^{3}$ The opposite is true of acute relapse.

In addition, it is not clear whether the sample was drawn from acute or long-stay wards and whether it consisted of patients who were stable on psychotropic medication and had insight or were acutely unwell. It is quite possible that most of the sample were patients who were stabilised on a drug regime, had insight and knew the purpose of their psychotropic medication. However, this may not be the case in acute episodes of care where the patient often lacks insight and questions the need to continue psychotropic medications. The authors state that in case of participants on more than two psychotropics, the 'longest-term treatment option' was selected. We fail to understand how this was established if duration of treatment was unknown. In the example given of a patient with bipolar disorder, the mood stabiliser was selected rather than the antipsychotic as the primary treatment; this was based on the assumption that mood stabilisers had been used first. However, it is well known that many patients are treated with antipsychotics as first-line medication. It is quite 
possible that antipsychotic medication was the initial intervention used and the patient took it as a matter of routine.

In summary, medication adherence is a complex issue that can be affected by various factors, such as lack of insight, religious and cultural beliefs, level of education and socioeconomic status, comorbid alcohol misuse, to name a few. ${ }^{4}$ We believe further studies are needed in this area.

1 Perecherla S, Macdonald AJD. Older psychiatric in-patients' knowledge about psychotropic and non-psychotropic medications. Psychiatrist 2011; 35: 220-4

2 Mitchell AJ, Selmes T. Why don't patients take their medicine? Reasons and solutions in psychiatry. Adv Psychiatr Treat 2007; 13: 336-46.

3 Droulout T, Liraud F, Verdoux H. Relationships between insight and medication adherence in subjects with psychosis. Encephale 2003; 29: 430-7.

4 Patel MX, David AS. Medication adherence: predictive factors and enhancement strategies. Psychiatry 2007; 6: 357-61.

Gurinder P. Singh, CT2 psychiatry, Health Lane Hospital, West Bromwich, Black Country Partnership NHS Foundation Trust, email: gurinder.singh@bcpft.nhs.uk; Aparna Prasanna, consultant old age psychiatrist, Black Country Partnership NHS Foundation Trust, Wolverhampton.

doi: $10.1192 / p b .36 .5 .195 a$

Authors' response: We agree that adherence to medication is important and subject to complex influences. We thought that understanding of medication was a neglected factor and set out to study this rather than adherence. We had hoped that this was clear. We were surprised to find that, broadly speaking, patients understood psychotropic and nonpsychotropic medication to the same degree. We confirm that patients from ethnic minorities who were able to speak English were included; patients were in acute wards and not long-stay wards (of which we have none). In the example of how we chose which medication to ask about, we do not say that we selected the mood stabiliser over the antipsychotic because it was given first. We chose it because it was likely to be used for the longest time. We agree that our sample was not representative of all older psychiatric patients and say as much in the discussion.

Sri Perecherla is consultant psychiatrist at Woodcote House, Croydon University Hospital, email: sri.perecherla@slam.nhs.uk. Alastair J. D. Macdonald is visiting professor of psychiatry in the Health Services and Population Research Department, Institute of Psychiatry, London.

doi: $10.1192 / \mathrm{pb} .36 .5 .196$

\section{Recruitment in psychiatry}

Those concerned about the dearth of young doctors applying to train as consultant psychiatrists might usefully consider the motives of those who make this choice. I am a recently retired general adult consultant psychiatrist that worked in England. I chose to undertake training because I wished to emulate senior consultants whom I met while acting as a medical student or junior doctor. I admired their determination and aspiration to improve the lives of those suffering from serious mental illness and their central role in the clinical care of those referred to mental health services. However, I fear junior doctors will now find it difficult to meet such inspirational and dynamic clinicians.

In England the blame culture consequent on the repeated internal, coroner and external enquiries, reconfiguration of services, the provisions of the amended Mental Health Act and New Ways of Working for consultants psychiatrists (and others) have all undermined morale. This last development left me without responsibility for my in-patients, the autonomy to arrange urgent admission when I thought this necessary or, in some cases, to refer for appropriate psychological therapy. Working became an increasing challenge. Our junior doctors notice these developments and their effect on senior colleagues' attitudes. It does not surprise me that the number opting to train remains worryingly low.

Keith E. Dudleston, consultant psychiatrist (retired), Ivybridge, email: tp@rcpsych.co.uk.

doi: $10.1192 / p b .36 .5 .196 a$

\section{Retaining trainees in psychiatry through a more mindful practice}

Barras \& Harris's survey on retention difficulties in psychiatry ${ }^{1}$ in provoking further discussion about the state of psychiatric training is an important piece of work. The systemic effects of the current economic crisis cannot be separated out from implications to services and in turn their impact is felt by those working and training within the system. Having myself recently completed higher training in psychotherapy, and through my experience of facilitating trainee case-based discussion groups, many of the trainees' comments picked up by Barras \& Harris felt all too familiar.

In terms of trainee concerns over the attitude of others towards psychiatry, I very much agree with the thinking of the authors that better integration of psychiatry with other specialties may increase understanding of both the contribution of psychiatry and challenge of mental health difficulties. Alongside this, I also think it is important to recognise that to bear with the projected 'madness' of others, which may mean we are seen as unsettling and to be kept a distance from perhaps by devaluation, is an important function of psychiatrists. Trainees' function as containers can be fostered, for example, in case-based discussion groups, enabling them to begin to understand and tolerate some of these processes as they are played out in their day-to-day work.

In Barras \& Harris's study, when asked about work and patient care, trainees complained about too much paperwork and a pressure to appear to be 'doing things correctly', which both undermine the real patient care. The concept of social defence, as described by Menzies-Lyth in her study of poor medical nursing staff retention in hospitals, ${ }^{2}$ is helpful in thinking about some of these difficulties. In mental hospitals, working practices which reduce contact with patients, such as the care of an individual patient being split into tasks or reduplication of checks to eliminate or share the responsibility of decisions, are used by staff/managers because of a fear of being in contact both with patients' and their own 'mad violence' and fragmentation. Further to this, the additional pressures of restructuring may both add to and be part of the same process. Ballatt \& Campling in Intelligent Kindness ${ }^{3}$ remind us that 'there is certainly evidence that major structural change keeps senior managers and board members detached from the front line of healthcare' (p. 131). In the face of this poor containment by the organisation, it is not surprising that morale is low among trainees. 